\title{
Conjecturas
}

\section{Aspectos morfofuncionais diafragmáticos e função pulmonar em pacientes pós covid-19 que foram submetidos a VMI}

\section{Diaphragmatic morphofunctional aspects and pulmonary function in post-covid-19 patients who have been submitted to invasive mechanical ventilation}

\author{
Maurício Rodrigues Comin ${ }^{1 *}$, Paulo Henrique Muleta Andrade ${ }^{1}$, Thamara Ferro Balsani Comin ${ }^{1}$, Rayssa \\ Bruna Holanda Lima ${ }^{1}$, Giseli Mayuki Ikeda Muleta Andrade ${ }^{2}$, Marília Rodrigues Leite de Castro ${ }^{3}$, Talita \\ Corrêa dos Santos ${ }^{4}$
}

\section{RESUMO}

Introdução: Os pacientes com COVID-19 comumente necessitam da utilização do suporte ventilatório por insuficiência respiratória aguda. Entretanto, a utilização de ventilação mecânica invasiva está relacionada a várias complicações. Além disso, há alta taxa de sequelas motoras, pulmonares, cardíacas e emocionais que ainda não estão bem esclarecidas na literatura. Objetivo: Avaliar e correlacionar os aspectos morfofuncionais diafragmáticos e função pulmonar de pacientes acometidos por COVID-19. Materiais e Métodos: Estudo transversal prospectivo que foi realizado no CER APAE de Campo Grande (MS), com aprovação do comitê de ética e pesquisa sob número: 5.004.510 em 28/09/2021. Os participantes foram avaliados através do exame de ultrassonografia diafragmática, também é mensurada a capacidade vital lenta, a capacidade expiratória forçada e a capacidade expiratória forçada em 1 segundo, contemplando os exames de função pulmonar (' e espirometria). Para avaliação subjetiva da fadiga foi utilizado o pictograma de fadiga, com objeto de correlacionar esses achados com os dados de função pulmonar e morfofuncionalidade diafragmática. Os participantes ajustam-se em dois grupos, conforme o tempo de alta hospitalar: menor ou igual a três meses de alta (grupo A classificado como síndrome pós COVID-19 aguda) e maior do que três meses (grupo B classificado como síndrome pós COVID-19 crônica). Resultados: foram avaliados 10 homens e 10 mulheres com média de idade de $41 \pm 7$ no grupo agudo e $51,5 \pm 4$ no grupo crônico com valor de $p<0,05$. Observou-se correlação positiva significativa com valor de $\mathrm{p}<0.05$ para as variáveis IMC e peso, espessura inspiratória diafragmática e espessura expiratória diafragmática, capacidade vital lenta e velocidade de contração, pictograma de fadiga domínio 1 e pictograma de fadiga domínio 2. Conclusão: A fadiga, a falta de ar e o cansaço foram as sintomatologias mais relatadas, entretanto, a maioria apresentou função pulmonar e morfologia diafragmática preservadas. Assim os fatores psicossomáticos, evidenciados pelo pictograma de fadiga, e a obesidade podem ser fatores que interferem negativamente na reabilitação desdes pacientes.

Palavras-chave: COVID-19; Ultrassonografia; Diafragma; Função pulmonar.

\footnotetext{
${ }^{1}$ Universidade Federal de Mato Grosso do Sul - UFMS

*E-mail: mauriciorcomin@icloud.com

${ }^{2}$ Tribunal de Justiça do Mato Grosso do Sul - TJMS

${ }^{3}$ Centro Especializado em Reabilitação da Associação de Pais e Amigos dos Excepcionais de Campo Campo Grande - Mato Grosso do Sul: CER APAE

${ }^{4}$ Instuição de afiliação. Centro Universitário UNIGRAN Capital - Campo Grande - MS
} 


\begin{abstract}
Introduction: Patients with COVID-19 commonly require the use of ventilatory support for acute respiratory failure. However, the use of invasive mechanical ventilation is related to several complications. In addition, there is a high rate of motor, pulmonary, cardiac and emotional sequelae that are still not well clarified in the literature. Objective: To evaluate and correlate diaphragmatic morphofunctional aspects and lung function in patients affected by COVID-19. Materials and Methods: Prospective cross-sectional study carried out at CER APAE in Campo Grande (MS), with approval by the ethics and research committee under number: 5,004,510 on 09/28/2021. Participants were evaluated through diaphragmatic ultrasound, slow vital capacity, forced expiratory capacity and forced expiratory capacity in 1 second are also measured, including pulmonary function tests (ventilometry and spirometry). For the subjective assessment of fatigue, the fatigue pictogram was used, with the aim of correlating these findings with data on pulmonary function and diaphragmatic morphofunctionality. Participants fit into two groups, according to the time of hospital discharge: less than or equal to three months of discharge (group A classified as acute post-COVID-19 syndrome) and greater than three months (group B classified as post-COVID syndrome -19 chronic). Results: 10 men and 10 women were evaluated with a mean age of $41 \pm 7$ in the acute group and $51.5 \pm 4$ in the chronic group, with $p<0.05$. There was a significant positive correlation with $\mathrm{p}$-value $<0.05$ for the variables BMI and weight, diaphragmatic inspiratory thickness and diaphragmatic expiratory thickness, slow vital capacity and contraction velocity, domain 1 fatigue pictogram and domain 2 fatigue pictogram. Conclusion: A fatigue, shortness of breath and tiredness were the most reported symptoms, however most had preserved lung function and diaphragmatic morphology. Thus, psychosomatic factors, evidenced by the fatigue pictogram, and obesity can be factors that negatively interfere in the rehabilitation of these patients.
\end{abstract}

Keywords: COVID-19; Ultrasonography; Diaphragm; Lung function.

\title{
INTRODUÇÃO
}

A COVID-19 é uma doença infecciosa, de alto contágio e elevada mortalidade, causada por um vírus denominado SARS-CoV-2. Os primeiros casos foram originários da cidade de Wuhan na China em novembro de 2019, declarado surto logo em janeiro de 2020, e posteriormente pandemia devido sua rápida e incontrolada disseminação. A maior parte dos primeiros contaminados estiveram expostos ao mercado de animais silvestres de Wuhan. Ainda, não se sabe a exata origem deste vírus, mas acredita-se que os morcegos possam ser uma possível fonte (ISLAM; IQBAL, 2020).

Atualmente foram confirmados 371.596.593 casos de COVID-19 no mundo. Deste números os países com maior incidência são: Estados Unidos com 49.387.208, seguido pela Índia com 34.656.822, Brasil 22.157.726, Reino Unido 10.620.535 e Rússia com 9.692.411. No que diz respeito aos óbitos, confirmaram-se ao redor do mundo 5.653.385 até o dia vinte e nove de janeiro de 2022, neste ranking o EUA lideram, seguidos pelo Brasil, Índia, México e Rússia respectivamente (Ministério da Saúde, 2022).

Após estudos sobre o novo vírus foi descoberto que pertence à família dos betacoronavírus, incluindo neste grupo o SARS-CoV e outros vírus da Síndrome Respiratória do Oriente 
Médio - MERS. O diagnóstico do novo coronavírus, após a evolução científica sobre a doença ocorre através do RT-PCR, padrão ouro, exame este que detecta o RNA do SARS-CoV-2 na amostra do paciente. Entretando várias outras técnicas foram criadas para tentar uma rápida deteccção do vírus, afim de proporcionar um rápido diagnóstico, evitando complicações (TALEGHANI; TAGHIPOUR, 2021).

Os pacientes com coronavírus podem apresentar sintomas severos de tosse, febre, falta de ar, fadiga e radiografia de tórax sugestiva de pneumonia (gravidade, internação, sindrome respiratoria aguda). A gravidade da COVID-19 e possível necessidade de leitos em centros de terapia intensiva tem sido o principal desafio para os governantes durante a pandemia, com a necessidade de ampliação e abertura de centros intensivos específicos para estes pacientes (YOUNG; THONE; JIK, 2020).

Hoje a COVID-19 é considerada uma patologia sistêmica, ou seja, afetando inúmeros tecidos e sistemas do corpo humano, mas os pulmões são os orgões mais acometidos e relacionados a maior gravidade e mortalidade desta doença. A experiência clínica até agora indica que o desenvolvimento da COVID-19 é bastante heterogêneo, podendo ser assintomatica, leve e grave. Os fatores de maior gravidade são: condições do próprio hospedeiro incluindo a idade, sexo e comorbidades como a diabetes, hipertensão arterial sistêmica e obesidade (BABAPOORFARROKHRAN et al., 2020).

Os pacientes que apresentam a forma grave da COVID-19 desenvolvem hipoxemia severa em decorrência do comprometimento pulmonar extenso, podendo evoluir para a Síndrome do Desconforto Respiratório Agudo (SDRA). Os cuidados intensivos na fase aguda do COVID-19 envolvem a possível necessidade da utilização de oxigênio suplementar e em casos de insuficiência respiratória aguda grave medidas mais invasivas com a intubação orotraqueal e instituição do suporte ventilatório invasivo (DHONT et al., 2020).

A SDRA causada pela COVID-19 é uma condição de alta mortalidade, a posição prona tem sido um metodo de escolha para o tratamento desta condição e vários ciclos de prona podem ser essenciais, associado a uso de altas doses de sedativos e em alguns casos de bloqueadores neuromusculares. A internação prolongada na unidade de terapia intensiva e hospitalar é a realidade da maioria dos pacientes que evoluem com a forma grave da COVID-19 (PETRONE; BRATHWAITE; JOSEPH, 2020). 
Sobre essa internação hospitalar prolongada, esta relacionada a inúmeras consequências relacionadas a redução da qualidade de vida pós alta hospitalar em decorrência do uso de sedativos e respiração artificial, imobilidade no leito, fraquezas musculares e redução da capacidade funcional, além da consequência da COVID-19 para a saúde a longo prazo. Dados de um estudo trouxe que seis meses após a infecção aguda pela COVID-19 os pacientes ainda apresentam fadiga e fraquezas musculares, dificuldades para dormir, ansiedade e quadros depressivos (HUANG et al., 2021).

Pacientes que apresentaram a SDRA pela COVID-19 podem apresentar sequelas como capacidade de difusão pulmonar reduzida e ainda comprometimentos pulmonares observados na radiografia pulmonar, sendo considerados a população alvo para a reabilitação a longo prazo (HUANG et al., 2021).

A espirometria é um teste de esforço pulmonar considerada uma das principais ferramentas para avaliar a aptidão respiratória. É capaz de quantificar a capacidade vital lenta (que é a soma do volume de reserva inspiratório, volume corrente e volume de reservar expiratório), além do volume minuto, que em outras palavras, nada mais é que a quantidade de ar que o paciente respira por um minuto. Esses valores em pacientes vítimas de infecção por coronavírus possuem a tendência de ser inferiores à normalidade, pois podem apresentar dificuldades em expandir o tecido pulmonar devido a lesões por este patógeno (POLICARPO BARBOSA et al., 2020).

Ainda sobre a quantificação da função pulmonar, outro exame padrão ouro para identificar disfunções, restritivas (dificuldade de gerar volume), obstrutiva (dificuldade em eliminar volume) e ou mista (soma das duas) é a espirometria. Os pacientes com sequelas pós COVID-19 costumam apresentar algumas anomalias, como diminuição da capacidade pulmonar total, volume expiratório forçado em 1 segundo subnormal, assim como a capacidade vital forçada. Vale ressaltar que alguns pacientes que receberam alta apresentam alterações residuais nas tomografias computadorizadas (TC) do tórax, com opacidade em vidro fosco como o padrão mais comum (RAMANATHAN et al., 2020).

Conforme Mo et al., 2020, dos 110 casos de COVID-19 de seu estudo, 27\% apresentaram capacidade pulmonar total diminuída; $13,6 \%$ com volume expiratório forçado inferior ao normal,; $9,1 \%$ com capacidade vital forçada diminuída, ainda, 67 dos casos configuraram com semelhança à pneumonia, 19 casos com pneumonia severa, observando-se: frequência respiratória superior a 30 incursões por minuto e saturação de oxigênio igual ou inferior à 93\%. 
O longo tempo de internação, associado a prolongamento da ventilação mecênica, como já citado, pode ocasionar a disfunção diafragmática - DD, e pode ser diagnosticada através da ultrassonografia cinesiológica, um método de avaliação confiável, acessível e não invasivo. No entanto, os critérios diagnósticos para a DD ainda não foram totalmente padronizados (MOJOLI et $a l ., 2019)$. A utilidade da ultrassonografia, para diagnóstico e qualidade do atendimento em pacientes internados nos centros de terapia intensivos gerais, são amplamente estudados na literatura, porém ainda são escassos os estudos específicos em centros de reabilitação fora hospitalar (SACHIN et al., 2020); (TURTON; ALAIDAROUS; WELTERS, 2019).

Os pacientes com síndrome pós COVID-19 podem apresentar sintomas como fadiga, cansaço, dispneia, fraqueza muscular, taquicardia, parestesia, perda ou ausência de olfato e paladar, cefaleia, distúrbios visuais e gastrointestinais, desequilíbrios e distúrbios emocionais. Tornando o tratamento complexo e multifatorial. Este estudo avaliou os aspectos morfofuncionais do músculo diafragma e as funções respiratórias, para poder identificar quais os fatores que poderiam interferir na recuperação e reabilitação pós COVID-19, auxiliando em um melhor tratamento, além de auxiliar e direcionar tomada de decisões da equipe multiprofissional.

A hipótese da presente pesquisa é que os participantes com alterações da espessura diafragmática e mobilidade diafragmática apresentam disfunção da função pulmonar, acarretando em uma recuperação mais demorada. Logo, tem como objetivo avaliar a relação entre as características morfofuncionais do músculo diafragmático e função pulmonar de pacientes pós COVID-19 que foram submetidos a ventilação mecânica invasiva.

\section{METODOLOGIA}

Estudo observacional, transversal, de caráter prospectivo, análitico e descritivo. A pesquisa foi realizada no Centro Especializado em Reabilitação da Associação de Pais e Amigos dos Excepicionais de Campo Grande - MS, CER APAE, no Ambulatório Sars-Cov-2. A amostra foi por conveniência. Participaram do estudo pessoas com disfunção pós-COVID-19, de ambos os sexos, no período de outubro de 2021 a dezembro de 2021, e que respeitaram os critérios de inclusão/não inclusão estabelecidos na presente pesquisa. 
Os participantes foram triados durante avaliação no ambulatório Sars-Cov-2 do CER APAE. E alocados em dois grupos: Grupo A (síndrome pós-COVID-19 aguda), com sintomas presistentes até três meses após alta hospitalar; e Grupo B (síndrome pós-COVID-19 crônica), com sintomas presistentes além de três meses após alta hospitalar. Foi coletado dados sociodemográficos, como idade, sexo, peso, queixas e comorbidades. Logo após foi realizado a ventilometria, espirometria, ultrassonagragia cinesiológica diafragmática e aplicação do pictograma de fadiga em um único momento.

Vale ressaltar que devido ao atual contexto de pandemia referente ao Coronavírus todos os cuidados foram redobrados, a fim de evitar contaminação. Foram seguidas todas as recomendações por parte do Ministério da Sáude e demais autoridades de saúde.

A avaliação morfofuncional diafragmática foi realizada utilizando-se ultrassom portátil (Mindray DP-30), contendo dois transdutores convexo (frequência: 2-6 MHz) e linear (frequência: 6-13MHz) no ambulatório Sars-Cov-2. (BOUSSUGES; GOLE; BLANC, 2009).

A espessura do músculo diafragma foi avaliada no modo $\mathrm{B}$ do ultrassom, com o transdutor linear posicionado entre o $8^{\circ}$ e $9^{\circ}$ espaço intercostal e na linha axilar direita. Assim que visualizado imagem clara, esta foi gravada em arquivo digital. A Fração de Espessamento do Músculo Diafragma (DTF) foi mensurada em três ciclos respiratórios, durante sua fase de relaxamento, ou seja, no final da fase expiratória. A fórmula do DTF utilizada foi: (espessura ao final da inspiração - espessura ao final da expiração) / espessura ao final da expiração X 100) (THIMMAIAH; MJ; JAIN, 2016). (RICOY et al., 2019).

A técnica utilizada para obtenção da mobilidade diafragmática foi executada no Modo M, pelo qual é possível observar estruturas que se movimentam. O transdutor utilizado foi o convexo, posicionado entre a linha paraesternal e a linha axilar anterior com uma frequência baixa $(2-6 \mathrm{MHz})$. Para a medição da amplitude de excursão, foi observado o ponto mais alto da inspiração (SANTANA et al., 2016)

A análise do capacidade vital lenta, volume minuto e cálculo do IRRS (FR/Volume corrente) foi avaliado com auxílio da ventilometria. O ventilômetro analógico utilizado foi do modelo Wright Mark-8- Marca Ferraris - Europa/Reino Unido. A técnica teve duração de no máximo dez (10) minutos, realizadas três (3) aferições para capacidade vital lenta, com descanso de um (1) minutos entre cada avaliação; e uma (1) aferição para obtenção do volume minuto (Rodrigues et al, 
2011). Durante toda a avaliação foi acompanhado o estado clínico do participante. (PINHEIRO FILHO et al., 2010).

A mensuração da capacidade vital (CV), capacidade vital forçada expiratória (CVF), o voulme expiratório forçado no primeiro segundo (VEF1) e a relação entre VEF1 e CVF, foi realizada utilizando-se o espirômetro digital da marca MIR, modelo Spirobank II. (RODRIGUES et al., 2011).

O pictograma de fadiga, é um instrumento que possui algumas figuras que avaliam o quanto a fadiga está impactando as atividades de vida diárias dos pacientes; é breve e de fácil aplicabilidade. Simplesmente o participante irá apontar ou marcar um "x" com uma caneta na imagem que o mesmo se identifica (MOTA; PIMENTA; FITCH, 2009).

Figura 1: Pictograma de fadiga.

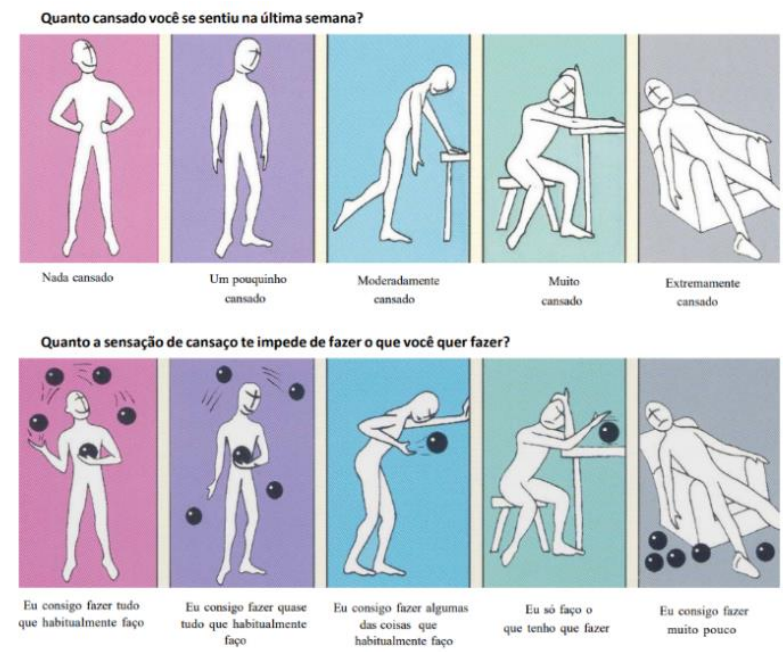

Fonte: (MOTA; PIMENTA; FITCH, 2009)

\section{RESULTADOS}

Foram avaliados 20 participantes, dez homens e dez mulheres, alocados em dois grupos conforme tempo de alta hospitalar.

Posto que a significância é para valor de P menor que 0,05 , e a hipótese nula do teste conduz à igualdade dos resultados entre os grupos, vê-se na tabela 1 - Distribuição de frequências das características sociodemográficas nos dois grupos estudados - a idêntica distribuição de resultados em ambos os grupos na variável "COMORBIDADES", apresentando a maioria dos participantes $(70 \%)$ como portadores de alguma comorbidade. Em relação ao peso, apesar de 
similares, o grupo A apresentou média e desvio padrão maiores em relação ao grupo B; mas o IMC do grupo B teve desvio padrão um pouco acima do que em A. Na variável "SEXO”, a maioria dos participantes em A (60\%) eram mulheres, já em B (60\%), homens. Por fim, na variável "IDADE" ocorreu uma diferença estatisticamente significativa, isso quer dizer que a hipótese nula do teste pode ser rejeitada.

Tabela 1: Distribuição de frequência das características sociodemográficas nos dois grupos estudados.

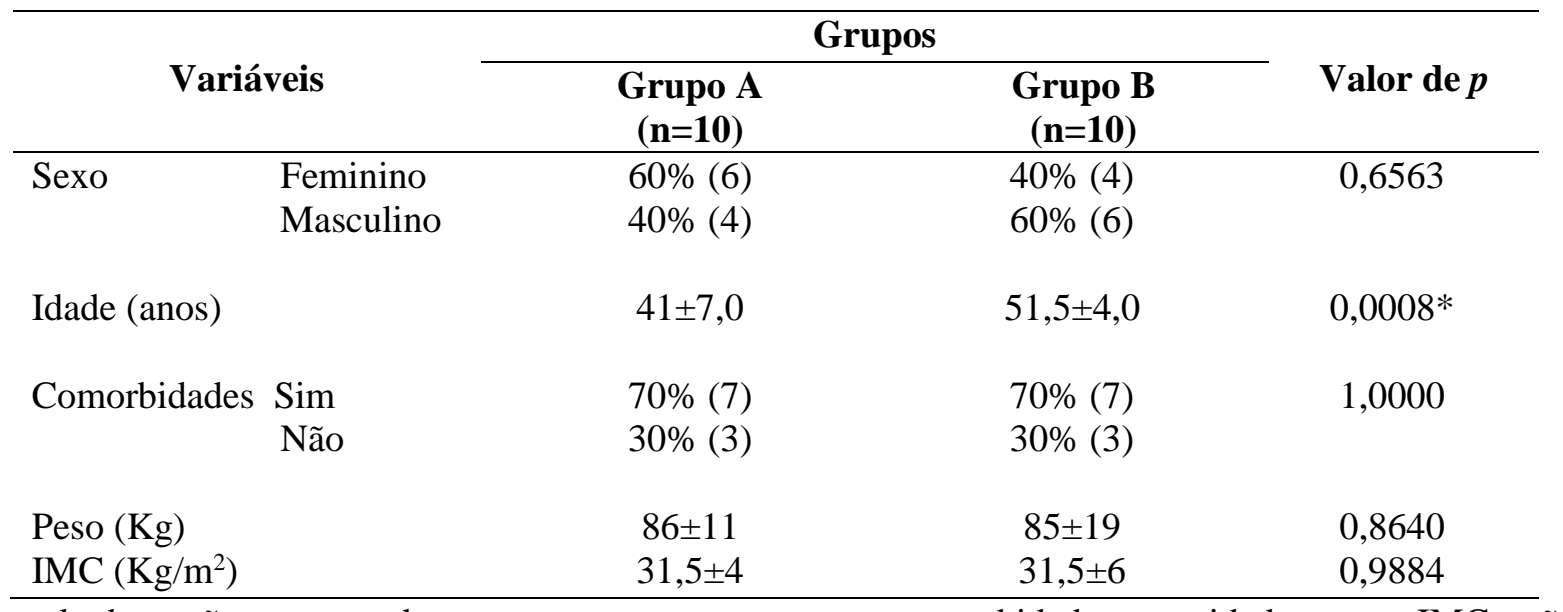

Os resultados estão apresentados em porcentagem para sexo e comorbidades; para idade, peso e IMC estão apresentados em média e desvio padrão. Teste t-student não pareado e exato de fisher. * Diferença estatisticamente significativa.

Na tabela 2 - Função pulmonar da população estudada - o laudo de espirometria indicou, para o grupo A, normalidade para a metade dos participantes, e a outra metade se distribuiu em grande parte (40\%) no DVR M, ou seja, ocorreu redução moderada da capacidade pulmonar total, e os $10 \%$ restantes apresentou redução grave da capacidade pulmonar total. Já para o grupo B a distribuição ocorreu mais equiparada, de forma que apenas $10 \%$ apresentou redução grave da capacidade pulmonar total, enquanto as demais variáveis - "NORMAL, DVR M e DVO M", sendo o último um distúrbio obstrutivo - contaram com o total de 30\% para cada. Quanto à capacidade vital lenta (CVL), ambos os grupos demonstraram redução de 80\% (grupo A) e 90\% (grupo B), isso significa a diminuição do volume corrente normal a cada ciclo respiratório do indivíduo. 
Tabela 2: Função pulmonar da população estudada.

\begin{tabular}{lcccc}
\hline \multirow{2}{*}{ Variável } & & \multicolumn{2}{c}{ Grupos } & \multirow{2}{*}{ Valor de P } \\
\cline { 3 - 4 } & & $\begin{array}{c}\text { Grupo A (n=10) } \\
\% \mathrm{n}\end{array}$ & $\begin{array}{c}\text { Grupo B (n=10) } \\
\% \mathrm{n}\end{array}$ & \\
\hline \multirow{2}{*}{ Laudo } & Normal & $50 \%(5)$ & $30 \%(3)$ & \multirow{2}{*}{0,3027} \\
Espirometria & DVR M & $40 \%(4)$ & $30 \%(3)$ & \\
& DVR G & $10 \%(1)$ & $10 \%(1)$ & \\
& DVO M & $0 \%(0)$ & $30 \%(3)$ & \\
CVL (ml) & & & & \\
& Normal & $20 \%(2)$ & $10 \%(1)$ & \\
& Reduzida & $80 \%(8)$ & $90 \%(9)$ & \\
\hline
\end{tabular}

Os resultados estão apresentados em porcentagem conforme laudos. DVR M = Distúrbio Ventilatório Restritivo Moderado; DVR G = Distúrbio Ventilatório Restritivo Grave; DVO M = Distúrbio Ventilatório Obstrutivo Moderado. Teste exato de Fisher e qui-quadrado.

Na tabela 3 - Avaliação ultrassonográfica diafragmática analisadas nos dois grupos - a maioria do grupo A (60\%) apresentou fração de espessamento diafragmático normal, enquanto 40\%, reduzida. Já o grupo B, teve $90 \%$ dentro da normalidade e apenas 10\% com DTF reduzida. Quanto à velocidade de contração, o grupo A obteve média maior que $\mathrm{B}$, porém menor desvio padrão. Para a variável "MOBILIDADE" os grupos contaram com médias e desvios padrão semelhantes, porém um pouco maiores em A, explicando o valor de P. Por último, as espessuras inspiratórias e expiratórias foram maiores no grupo A, sendo a principal diferença na espessura expiratória.

Tabela 3: Avaliação ultrassonográfica diafragmática analisadas nos dois grupos.

\begin{tabular}{cccc}
\hline \multirow{2}{*}{ Variável } & \multicolumn{2}{c}{ Grupos } & \multirow{2}{*}{ Valor de P } \\
\cline { 2 - 3 } & Grupo A (n=10) & Grupo B (n=10) & 0,3992 \\
Esp. INSP. & $0,2570 \pm 0,0827$ & $0,2270 \pm 0,0723$ & 0,3105 \\
Esp. EXP. & $0,2030 \pm 0,0764$ & $0,1700 \pm 0,0644$ & \\
$\quad(\mathrm{~cm})$ & $2,1830 \pm 1,0730$ & $2,1170 \pm 1,0420$ & 0,8110 \\
Mobilidade & $1,393 \pm 1,083$ & 0,4925 \\
Vel. Contração & $1,4780 \pm 1,0750$ & & \\
& & $90 \%(9)$ & 0,3034 \\
DTF Normal & $60 \%(6)$ & $10 \%(1)$ & \\
DTF Reduzida & $40 \%(4)$ & par & \\
\hline
\end{tabular}

Os resultados estão apresentados em média e desvio padrão para a espessura inspiratória, espessura expiratória, mobilidade e velocidade de contração. Já para fração de espessamento diafragmática (DTF) dados representados em porcentagem conforme diagnóstico. Teste t-student não pareado, teste de Mann-Whitney (mobilidade e velocidade de contração) e teste exato de fisher. 
Na tabela 4 - Avaliação dos domínios do pictograma de fadiga para a população estudada -, na variável "DOMÍNIO 1", ambas as metades dos grupos A e B pontuaram sentir-se "um pouquinho cansado" na última semana. No grupo A não houveram indicações para "nada cansado" nem "extremamente cansado", porém três sentiam-se moderadamente cansados e dois, muito cansados. Já no grupo B, não houveram indicações de sentir-se "extremamente cansado" e apenas um indicou não estar nada cansado; os demais afirmaram estar moderadamente cansados (3) e muito cansado (1). Para a variável "DOMÍNIO 2" metade do grupo A afirmou ser capaz de fazer algumas das coisas habituais; três afirmaram fazer tudo normalmente, um faz muito pouco e um faz quase tudo do seu habitual. No grupo B a maioria faz quase tudo do seu habitual, dois fazem apenas algumas coisas, um faz tudo que está habituado e um faz apenas o que necessário.

Tabela 4: Avaliação dos domínios do pictograma de fadiga para a população estudada.

\begin{tabular}{llccc}
\hline \multirow{2}{*}{ Domínio } & & \multicolumn{2}{c}{ Grupos } & \multirow{2}{*}{ Valor de P } \\
\cline { 2 - 4 } & 0 & $\begin{array}{c}\text { Grupo A } \\
(\mathbf{n = 1 0})\end{array}$ & $\begin{array}{c}\text { Grupo B } \\
(\mathbf{n = 1 0})\end{array}$ & \\
\hline \multirow{3}{*}{ Domínio 1 } & $(0)$ & $(1)$ & \\
& 1 & $(5)$ & $(5)$ & 0,7212 \\
& 2 & $(3)$ & $(3)$ & \\
& 3 & $(2)$ & $(1)$ & \\
Domínio 2 & 4 & $(0)$ & $(0)$ & 0,0970 \\
& 0 & $(3)$ & $(1)$ & \\
& 1 & $(1)$ & $(6)$ & \\
& 2 & $(5)$ & $(2)$ & \\
& 3 & $(0)$ & $(1)$ & \\
& 4 & $(1)$ & $(0)$ & \\
\hline
\end{tabular}

Os resultados estão apresentados em quantidade de participantes que assinalaram as respectivas respostas. Domínio 1 (quanto cansado você se sentiu na última semana?): 0 = nada cansado; 1 = um pouquinho cansado; 2 = moderadamente cansado; 3 = muito cansado; 4 = extremamente cansado. Domínio 2 (quanto a sensação de cansaço te impede de fazer o que você quer fazer): $0=$ eu consigo fazer tudo que habitualmente faço; $1=$ eu consigo fazer quase tudo que habitualmente faço; $2=$ eu consigo fazer algumas das coisas que habitualmente faço; 3 = eu só faço o que tenho que fazer; 4 =eu consigo fazer muito pouco. Teste quiquadrado.

Conforme a tabela 5, correlação matrix das variáveis estudadas em todos os pacientes do estudo. Foi observado correlação positiva significativa com valor de $\mathrm{p}<0.05$ para as variáveis IMC/peso, espessura inspiratória diafragmática/espessura expiratória diafragmática, capacidade vital lenta/velocidade de contração, pictograma de fadiga domínio 1/pictograma de fadiga domínio 2 . Isso significa que quando um aumenta o outro segue nesta mesma linha proporcionalmente. Já as variáveis IMC/mobilidade diafragmática e peso/mobilidade diafragmática apresentaram correlação negativa significativa com valor de $\mathrm{p}<0.05$, logo interpretamos que quanto maior for uma variável menor será a correspondente. 
Tabela 5: Análises de correlação das variáveis estudadas de pacientes com síndrome pós-COVID-19.

\begin{tabular}{|c|c|c|c|c|c|c|c|c|c|c|c|}
\hline & Idade & IMC & Peso & Vent. CVL & Vent. VE & Esp. Insp. & Esp. Exp. & Mobilidade & Vel. Contração & PIC 01 & PIC 02 \\
\hline Idade & & $-0,043$ & $-0,057$ & $-0,419$ & 0,228 & $-0,073$ & $-0,083$ & $-0,005$ & $-0,286$ & $-0,078$ & 0,093 \\
\hline IMC & & & 0,845 & $-0,071$ & 0,256 & 0,214 & 0,159 & $-0,607$ & $-0,315$ & 0,287 & 0,120 \\
\hline Peso & & & & 0,156 & 0,293 & 0,296 & 0,281 & $-0,440$ & $-0,054$ & 0,278 & 0,092 \\
\hline Vent CVL & & & & & 0,249 & 0,139 & 0,175 & 0,245 & 0,450 & $-0,266$ & $-0,074$ \\
\hline Vent VE & & & & & & $-0,177$ & $-0,273$ & $-0,135$ & 0,012 & $-0,361$ & $-0,357$ \\
\hline Esp. Insp. & & & & & & & 0,962 & $-0,093$ & $-0,054$ & 0,220 & 0,105 \\
\hline Esp. Exp. & & & & & & & & $-0,047$ & 0,029 & 0,246 & 0,167 \\
\hline Mobilidade & & & & & & & & & 0,356 & $-0,092$ & 0,035 \\
\hline Vel. Contração & & & & & & & & & & $-0,281$ & $-0,122$ \\
\hline PIC 01 & & & & & & & & & & & 0,544 \\
\hline PIC 02 & & & & & & & & & & & \\
\hline
\end{tabular}

Os resultados estão apresentados em valor de "r", com aplicação do teste de Correlação de Spearman para idade, índice de massa corporal, peso, ventilometria - capacidade vital lenta, ventilometria - volume minuto, espessura inspiratória, espessura expiratória, mobilidade, velocidade de contração, pictograma de fadiga domínio 1 e pictograma de fadiga domínio 2 . Quando $0<\mathrm{r}<0,25$ ou $-0,25<\mathrm{r}<0$ : correlação pequena ou nula; quando $0,25<\mathrm{r}<0,50$ ou $-0,50<$ $\mathrm{r}<-$ 0,25: correlação fraca; quando $0,50<\mathrm{r}<0,75$ ou $-0,75<\mathrm{r}<-0,25$ : correlação moderada; quando $0,75<\mathrm{r}<1,00$ ou $-1,00<\mathrm{r}<-075$ : correlação perfeita. Para uma correlação perfeita $r=-1$ ou $r=1$ 


\section{DISCUSSÃO}

A resposta imune ao vírus da COVID-19 inicia intensa liberação de citocinas e demais mediadores químicos, o que pode causar linfopenia, trombose e infiltração celular, levando os enfermos ao estado crítico (Cesar \& Mello, 2021.). A maioria dos pacientes do estudo apresentou como sequelas: hipertensão arterial sistêmica (HAS); alterações respiratórias pulmonares; fadiga; e também há a hipótese de o diabetes corresponder ao quadro pós-infecção. Relaciona-se aos resultados a entrada do vírus com auxílio da enzima convertedora de angiotensina II (ECA-II) ao estresse oxidativo decorrente da terapia de ventilação mecânica (VM) e ao desbalanço bioquímico gerado (ALCOCER-DÍAZ-BARREIRO et al., 2020).

A VM visa auxiliar pacientes críticos na manutenção das trocas gasosas e ritmo respiratório, com posterior retorno às funções fisiológicas. Entretanto, seu uso prolongado pode desencadear alterações bioquímicas (CRUZ et al., 2021), mormente devido ao estresse oxidativo, resultado da redução da atividade antioxidante, como a glutationa, permitindo a autofagia lisossomal. Outrossim, há elevação da proteólise: aumento dos níveis da proteína carbonila e da catalase, decompondo o peróxido de hidrogênio até ativação da via de degradação proteica ubiquitina proteossoma (Andrade, 2020). Tais fatores podem acarretar em doenças silenciosas, como as sequelas citadas, além dealterações no trofismo e disfunção contrátil do diafragma.

O surgimento de HAS após o COVID-19 pode ser decorrente das alterações a nível microscópico nos capilares cardíacos, ou da predisposição genética do indivíduo. Comprovadamente houve angiogênese intussusceptiva (REICHARDT et al., 2021) em um grupo de estudo na Alemanha, a qual pode ser relacionada à supracitada ECA-II auxilia no crescimento de novos vasos na musculatura e é um receptor do vírus. Então, essa ligação vírus-receptor, acarreta na superprodução da angiotensina II pelo ECA, induzindo à severa vasoconstrição e inflamação no tecido (LACERDA et al., 2014), explica o quadro de hipertensão arterial, junto ao emaranhado de capilares formado no tecido cardíaco, o qual pode ser um quadro crônico.

A fadiga crônica é relatada por alguns pacientes, no entanto, aponta-se relação com fatores psicossomáticos ao invés de alterações bioquímicas ou outros mediadores. Um estudo na Itália comprovou normalidade em pacientes que reportaram sintomas 
crônicos da COVID-19 (MANTOVANI et al., 2021). Avaliada a cadeia de luz de neurofilamentos, um biomarcador do axônio neural, não foi possível determinar a sua relação com a fadiga. Como os demais exames de ultrassonografia cinesiológica e espirometria apresentaram resultados próximos à normalidade - entende-se que pode ser um sintoma neuropsiquiátrico que pode estar relacionado com os traumas vividos e a qualidade alimentar e de sono.

Já sobre o diabetes, tal qual o HAS, pode ser decorrente da predisposição genética ou das alterações metabólicas causadas pelo vírus ou pela terapia medicamentosa. Muitos pacientes são tratados com glicocorticoides, podendo induzir a hiperglicemia, a qual também pode ocorrer devido ao estresse causado por infecções agudas. Ademais, com a ativação do sistema renina-angiotensina e da cascata imunológica propiciando a autoimunidade contra células $\beta$-pancreáticas (SATHISH; ANTON; SIVAKUMAR, 2021), há a hipótese de que o organismo desenvolva diabetes após alguns meses ou anos. Outro ponto a ser considerado é o fator emocional (elencado pela grande maioria dos participantes, especialmente decorrente das "alucinações" intrahospitalar) junto à má alimentação pré e pós internação (não tão saudável), desenvolvendo acúmulo de açúcares no organismo e consequente diabetes.

Em um estudo com o objeto de descrever as principais sequelas pós-COVID19, os autores afirmam que a fadiga, falta de ar, cansaço, fraqueza e distúrbios do sono são as mais persistentes e estão realacionada com fatores de risco, inclusive a idade, com sintomatologia crônica mais frequentes em pessoas com idade avançada. Assim como na presente pesquisa a média de idade mostrou-se mais elevada no grupo crônico em relação ao aguda, podendo ser justificada ao fato de que ao passar dos anos, a capacidade de recuperação e regeneração, assim como o porcesso de cura, correm de forma mais lenta, quando comparada com indivíduos mais jovens (RAMANATHAN et al., 2020).

A obesidade tem um impacto negativo na função pulmonar, na função muscular respiratória e na qualidade de vida, além de ser uma condição incapacitante. $\mathrm{O}$ treino pré-operatório desses indivíduos são extremamente importantes, visto que são comumente mais afetados por complicações pulmonares pós internações, seja por motivos cirúrgicos ou patólogicos, em especial ocorrendo a perda da capacidade de gerar volume pulmonar e força muscular respiratória, podendo serem classificados como "restritos" (TENÓRIO et al., 2013). Na avaliação ultrassonográfica pode-se observar 
redução da mobilidiade diafragmática com correlação de IMC e ou peso mais elevados, em ambos os grupos estudados, mostrando valores significativos e com valor de $p<0,05$ (ANJOS, 1992)

A ventilação mecânica invasiva e a internação prolongada estão associadas com sequelas, como perda de massa muscular, atrofia diafragmática, diminuição da complacência pulmonar, alterações cardiovasculares, além de inúmeros outros prejuízos sistêmicos. Ainda a infecção por COVID-19 pode acarretar em fibrose pulmonar pós internação, devido a evolução da reparação da lesão tecidual parenquimatosa (VERNEY BENICIO SILVA SÁ et al., 2021). Observou-se a presença de distúrbios respiratórios restritivos moderados e fração de espessamento do diafragma reduzida em ambos os grupos deste estudo, com prevalência em indivíduos classificados como agudos, podendo ser uma sequela da perda de complacência, desuso do músculo diafragma devido a VM como também por cicatrização tecidual pulmonar.

A ventilometria e a espirometria avaliam as capacidades funcionais ventilatórias e volumes pulmonares, e podem auxiliar de forma indireta a caracterização da efetividade do músculo diafragma (como exemplo a força, capacidade contrátil e resitência). Já a mobilidade, fração de espessamento e a velocidade de contração, são capazes de quantificar de forma direta a função e morfologia do músculo diafragma (GOTTESMAN; MCCOOL, 1997). A capacidade vital lenta correlacionou-se positivamente com a velocidade de contração diafragmática em ambos os grupos estudaddos, ou seja, quanto maior a CVL maior a velocidade contrátil do diafragma.

O Pictograma de Fadiga precisa de ajustes para pessoas sem doenças, mas mostra-se válido, confiável e fácil de usar para avaliar fadiga em pessoas com algum tipo de enfermidade crônica ou degenerativa (MOTA; PIMENTA; FITCH, 2009). A maioria dos participantes deste estudo relataram fadiga e cansaço, assim como ansiedade e distúrbios no sono, mostrando-se presente mesmo em aqueles com função pulmonar e morfologia diafragmática sem alterações significativas. Ainda, quanto maior foi a pontuação no domínio 1 (sensação de cansaço) maior foi a pontuação do domínio 2 (quando essa sensação de cansaço impede de fazer as atividades do dia a dia), mostrando uma correlação positiva.

A diminuição dos casos agudos da síndrome pós-COVID-19, visivelmente pela diminuição das triagens no setor correspondente, foi um fator importante para a 
redução do número da amostra. Este fato, felizmente ocorreu devido muito provavelmente pela primeira dose da vacina e suas devidas doses de reforço. Faz-se necessário novos estudos que comparem a funcionalidade pulmonar e morfologia diafragmática com teste ergométrico, com o intuito de esclarever fadiga física de fadiga emocional.

\section{CONCLUSÃO}

O índice de massa corpórea e o peso foram fatores contribuintes para determinar a redução da mobilidade diafragmática, assim a obesidade mostra-se um fator de risco para a síndrome pós-COVID-19. Ainda, a fadiga, a falta de ar e o cansaço foram as sintomatologias mais relatadas, entretanto a maioria apresentou função pulmonar e morfologia diafragmática preservadas, assim os fatores psicossomáticos, evidenciados pelo pictograma de fadiga, podem ser fatores que interferem negativamente na reabilitação desdes pacientes.

\section{REFERÊNCIAS}

ALCOCER-DÍAZ-BARREIRO, Luis et al. COVID-19 and the renin, angiotensin, aldosterone system. A complex relationship. Archivos de Cardiologia de Mexico, v. 90, p. 19-25, 2020.

ANJOS, Luiz A. Body mass index as a tool in the nutritional assessment of adults: a review. Revista de Saúde Pública, v. 26, n. 6, p. 431-436, 1992.

BABAPOOR-FARROKHRAN, Savalan et al. Myocardial injury and COVID-19: Possible mechanisms. Life Sciences, v. 253, n. April, p. 117723, 2020. Disponível em: <https://doi.org/10.1016/j.1fs.2020.117723>.

BOUSSUGES, Alain; GOLE, Yoann; BLANC, Philippe. Diaphragmatic motion studied by M-mode ultrasonography. Chest, v. 135, n. 2, p. 391-400, 2009. Disponível em: <http://dx.doi.org/10.1378/chest.08-1541>.

CESAR, Marcos; MELLO, Ramos. Ventilação mecânica. [S.l: s.n.], [S.d.].

CRUZ, Daniel Alves et al. Impactos da ventilação mecânica invasiva em pacientes de COVID-19: revisão integrativa. Research, Society and Development, v. 10, n. 11, p. e380101119656, 2021. 
DHONT, Sebastiaan et al. The pathophysiology of "happy" hypoxemia in COVID-19. Respiratory Research, v. 21, n. 1, p. 1-9, 2020.

GOTTESMAN, Eric; MCCOOL, F. Dennis. Ultrasound evaluation of the paralyzed diaphragm. American Journal of Respiratory and Critical Care Medicine, v. 155, n. 5, p. $1570-1574,1997$.

HUANG, Chaolin et al. 6-month consequences of COVID-19 in patients discharged from hospital: a cohort study. The Lancet, v. 397, n. 10270, p. 220-232, 2021. Disponível em: <http://dx.doi.org/10.1016/S0140-6736(20)32656-8>.

ISLAM, Khursheed Ul; IQBAL, Jawed. An Update on Molecular Diagnostics for COVID-19. Frontiers in Cellular and Infection Microbiology, v. 10, n. November, p. 1$11,2020$.

LACERDA, ONEIDE RAIANNY MONTEIRO et al. Artigo de Revisão Artigo de Revisão. Sociedade Brasileira de Cardiologia, v. 9, n. 1, p. 201-208, 2014.

MANTOVANI, Elisa et al. Chronic fatigue syndrome: an emerging sequela in COVID19 survivors? Journal of NeuroVirology, v. 27, n. 4, p. 631-637, 2021. Disponível em: <https://doi.org/10.1007/s13365-021-01002-x>.

MO, Xiaoneng et al. Abnormal pulmonary function in COVID-19 patients at time of hospital discharge. European Respiratory Journal, v. 55, n. 6, p. 2-5, 2020.

MOJOLI, Francesco et al. Lung ultrasound for critically ill patients. American Journal of Respiratory and Critical Care Medicine, v. 199, n. 6, p. 701-714, 2019.

MOTA, Dálete Delalibera Corrêa de Faria; PIMENTA, Cibele Andrucioli de Mattos; FITCH, Margaret Isabel. Pictograma de Fadiga: uma alternativa para avaliação da intensidade e impacto da fadiga. Revista da Escola de Enfermagem da USP, v. 43, n. spe, p. 1080-1087, 2009.

OXIDATIVO, Estresse; OXIDATIVO, Estresse. Andrade CS et al. Ventilação mecânica invasiva... PESQUISA Ventilação mecânica invasiva induzindo estresse oxidativo no diafragma: uma revisão de literatura. p. 1-9, 2020.

PETRONE, Patrizio; BRATHWAITE, Collin E.M.; JOSEPH, D'Andrea A.K. Prone ventilation as treatment of acute respiratory distress syndrome related to COVID-19. European Journal of Trauma and Emergency Surgery, 2020. Disponível em: <https://doi.org/10.1007/s00068-020-01542-7>.

PINHEIRO FILHO, Gilvan Reis et al. Comparison and effects of two different airway occlusion times during measurement of maximal inspiratory pressure in adult intensive care unit neurological patients. Revista Brasileira de terapia intensiva, v. 22, n. 1, p. 33-339, 2010.

POLICARPO BARBOSA, Fernando et al. Prediction of maximum oxygen uptake through incremental exercise testing using ventilometry: a cross-sectional study. Brazilian Journal of Physical Therapy, v. 24, n. 4, p. 365-372, 2020. Disponível em: <https://doi.org/10.1016/j.bjpt.2019.07.002>.

RAMANATHAN, Kollengode et al. 6-month consequences of COVID-19 in patients discharged from hospital: a cohort study. The Lancet, v. 397, n. January, p. 19-21, 
2020.

REICHARDT, Marius et al. 3D virtual Histopathology of Cardiac Tissue from Covid19 Patients based on Phase-Contrast X-ray Tomography. bioRxiv, p. 1-28, 2021.

RICOY, J. et al. Diaphragmatic dysfunction. Pulmonology, v. 25, n. 4, p. 223-235, 2019.

RODRIGUES, Alfredo José et al. Preoperative respiratory muscle dysfunction is a predictor of prolonged invasive mechanical ventilation in cardiorespiratory complications after heart valve surgery. European Journal of Cardio-thoracic Surgery, v. 39, n. 5, p. 662-666, 2011.

SACHIN, Sasidharan et al. Ultrasonographic evaluation of lung and heart in predicting successful weaning in mechanically ventilated neurosurgical patients. Journal of Clinical Monitoring and Computing, n. 0123456789, 2020. Disponível em: <https://doi.org/10.1007/s10877-020-00460-8>.

SANTANA, Pauliane Vieira et al. Identificação da diminuição da mobilidade diafragmática e do espessamento diafragmático na doença pulmonar intersticial : utilidade da ultrassonografia. J Bras Pneumol, v. 42, n. 2, p. 88-94, 2016.

SATHISH, Thirunavukkarasu; ANTON, Mary Chandrika; SIVAKUMAR, Tharsan. New-onset diabetes in "long COVID”. Journal of Diabetes, v. 13, n. 8, p. 693-694, 2021.

SAÚDE, Ministério Da. Boletim epidemiológico especial. Secretaria de Vigilância em Saúde, v. 61, p. 103, 2021.

TALEGHANI, Nastaran; TAGHIPOUR, Fariborz. Diagnosis of COVID-19 for controlling the pandemic: A review of the state-of-the-art. Biosensors and Bioelectronics, v. 174, p. 112830, 2021. Disponível em: <https://doi.org/10.1016/j.bios.2020.112830>.

TENÓRIO, Luís Henrique Sarmento et al. The influence of inspiratory muscle training on diaphragmatic mobility, pulmonary function and maximum respiratory pressures in morbidly obese individuals: A pilot study. Disability and Rehabilitation, v. 35, n. 22, p. 1915-1920, 2013.

THIMMAIAH, Vishwanath T; MJ, Geetha; JAIN, Keval P. Evaluation of Thickness of Normal Diaphragm by B Mode Ultrasound. International Journal of Contemporary Medical Research, v. 343, n. 9Online, p. 2393-915, 2016. Disponível em: <www.ijcmr.com>.

TURTON, Peter; ALAIDAROUS, Sondus; WELTERS, Ingeborg. A narrative review of diaphragm ultrasound to predict weaning from mechanical ventilation: where are we and where are we heading? Critical Ultrasound Journal, v. 11, n. 1, 2019. Disponível em: <https://doi.org/10.1186/s13089-019-0117-8>.

VERNEY BENICIO SILVA SÁ, Idalo et al. Reabilitação pulmonar pós-covid-19. Internacional Saúde Única (Interface Mundial), v. 47, n. 1, p. 10-12, 2021.

YOUNG, Jee; THONE, Melissa N; JIK, Young. Since January 2020 Elsevier has created a COVID-19 resource centre with free information in English and Mandarin on 
the novel coronavirus COVID- 19 . The COVID-19 resource centre is hosted on Elsevier Connect, the company' s public news and information. n. January, p. 25, 2020. Disponível em:

<https://www.ncbi.nlm.nih.gov/pmc/articles/PMC7759095/pdf/main.pdf〉.

Recebido em: 15/01/2022

Aprovado em: 16/02/2022

Publicado em: 18/02/2022 